Annals of Pure and Applied Mathematics

Vol. 12, No. 2, 2016, 233-238

ISSN: 2279-087X (P), 2279-0888(online)

Published on 18 December 2016

www researchmathsci.org

DOI: http://dx.doi.org/10.22457/apam.v12n2a14

\section{Annals of}

Pure and Applied

Mathematics

\title{
A Study on the Normed Graphs and Disemigraphs
}

\author{
Mathew Varkey T. $K^{1}$ and Susan Ray Joseph ${ }^{2}$ \\ ${ }^{1}$ Department of Mathematics, T.K.M.College of Engineering, Kollam-5, Kerala, India. \\ e-mail: mathewvarkeytk@gmail.com \\ ${ }^{2}$ Department of Mathematics, M.A.College, Kothamangalam-686 666, Kerala, India. \\ e-mail: susanrayjoseph@gmail.com
}

Received 22 November 2016; accepted 12 December 2016

Abstract. The norm analogue defined on graphs is known as normed graph. This paper is a study of normed graph of a disemigraph. We observe in this paper that a set of spanning subdisemigraphs with a norm function defined on the disemigraphs gives a normed graph associated with the consecutive adjacency graph of the disemigraph.

Keywords: Graph function, graph norm, normed digraph, spanning subsemigraphs

AMS mathematics subject classification (2010): 05E99

\section{Introduction}

The normed graphs defined in [7] is a digraph obtained by associating a norm function to a set of spanning subgraphs of a given graph. Semigraphs introduced by Sampathkumar [5] is a generalization of the concept of graphs in a natural way. Many have studied the properties of the semigraphs and have defined parameters like independence number, domination number, covering number etc. These parameters on spanning subsemigraphs are some real valued graph functions. Real valued functions defined on graphs satisfying the conditions of norm generates a new graph.

\section{Preliminaries}

The basic definitions used in this paper are from references $[1,3,5,6]$ given in last section.

Definition 2.1. [6] Any function defined on the set $\mathrm{S}$ of spanning subgraphs of a graph is called a graph function.

Definition 2.2. [6] A graph function g which satisfies the conditions of norm is said to be a graph norm.

Definition 2.3. [6] A graph function $g$ is said to be a graph norm if $g$ satisfies the norm conditions. That is, if $g: S \rightarrow R^{*}$ where $S$ is a collection of spanning subgraphs $\left\{H_{1}, H_{2}, \ldots . ., H_{n}\right\}$ of the graph $G$ and $\quad R^{*}=R^{+} U\{0\}, g$ satisfies

(i) $\quad g\left(H_{\mathrm{i}}\right) \geq 0, g\left(H_{\mathrm{i}}\right)=0 \Leftrightarrow H_{\mathrm{i}}$ is an empty graph.

(ii) $\quad g\left(H_{\mathrm{i}} \oplus H_{\mathrm{j}}\right) \leq g\left(H_{\mathrm{i}}\right)+g\left(H_{\mathrm{j}}\right)$ 
Mathew Varkey T.K and Susan Ray Joseph

(iii) $\quad g\left(\alpha H_{\mathrm{i}}\right)=\alpha g\left(H_{\mathrm{i}}\right)$ for every $i$ and $j, i \neq j, H_{\mathrm{i}}, H_{\mathrm{j}} \in S, \alpha \in R^{*}$. (where $\oplus$ denotes the ring sum of $H_{\mathrm{i}}$ and $H_{\mathrm{j}}$.)

On defining a norm $g$ on a set $S$ of spanning subgraphs of a graph $G$, a digraph called the relative normed graph $N_{G}(S)$ of $S$ on $G$ with respect to the graph norm $g$ is generated.

To construct $\mathbf{N}_{\mathbf{G}}(\mathbf{S})$. [6] The vertex set of $N_{G}(S)$ is $\mathrm{S}$ and a vertex $\mathrm{H}_{\mathrm{i}}$ is said to be adjacent to vertex $\mathrm{H}_{\mathrm{j}}$ if $g\left(H_{\mathrm{i}}\right) \leq g\left(H_{\mathrm{j}}\right)$ for every $\mathrm{i}$ and $\mathrm{j}, \mathrm{i} \neq \mathrm{j}$.

In this paper, we see the relative normed graph of $S$ on a semigraph and on a disemigraph $G$ with respect to a norm $g$ defined on $S$. The generated graph $N_{G}(S)$ is a digraph.

Definition 2.4. [6] If $D$ is a directed graph the graph $N_{D}(S)$ is said to be a normed graph if $D \cong N_{D}(S)$ for some collection $\mathrm{S}$ of spanning subdigraphs of $D$.

Definition 2.5. [6] The ring sum of two graphs $G_{1}$ and $G_{2}$ denoted by $G_{1} \oplus G_{2}$ is a graph consisting of the vertex set $V_{1} U V_{2}$ and the edges that are either in $G_{1}$ or $G_{2}$ but not in both. Then $G \oplus G=\phi$.

If $G_{1}$ and $G_{2}$ are edge disjoint then $G_{1} \oplus G_{2}=G_{1} U G_{2}$.

In [7] the concept of norm of a graph in two ways is considered, one, on the collection of spanning sub graphs of a graph and the other is on the collection of spanning sub graphs of a graph forming a vector space.

The collection of all spanning subgraphs of a graph $\mathrm{G}$ forms a linear space over $\mathrm{Z}_{2}$ under the ring sum operation of subgraphs and scalar multiplication of subgraphs. This linear space with a norm defined on it is called a graph normed space.

Definition 2.6. [5] A semigraph $G$ is a pair $(V, X)$ where $V$ is a nonempty set whose elements are called vertices of $G$ and $X$ is a set of n-tuples called edges of $G$ of distinct vertices for $\mathrm{n} \geq 2$ satisfying the following conditions.

(1) Any two edges have atmost one vertex in common.

(2) Two edges $\left(\mathrm{u}_{1}, \mathrm{u}_{2}, \ldots .,, \mathrm{u}_{\mathrm{n}}\right)$ and $\left(\mathrm{v}_{1}, \mathrm{v}_{2}, \ldots . ., \mathrm{v}_{\mathrm{m}}\right)$ are considered to be equal, if and only if,

(i) $\mathrm{m}=\mathrm{n}$ and

(ii) either $u_{i} \leq v_{i}$ for $1 \leq \mathrm{i} \leq \mathrm{n}$ or $\mathrm{u} \leq \mathrm{v}_{\mathrm{n}-\mathrm{i}+1}$ for $1 \leq \mathrm{i} \leq \mathrm{n}$.

Thus the edge $\left(\mathrm{u}_{1}, \mathrm{u}_{2}, \ldots . \mathrm{u}_{\mathrm{n}}\right)$ is the same as the edge $\left(\mathrm{u}_{\mathrm{n}}, \mathrm{u}_{\mathrm{n}-1}, \ldots, \mathrm{u}_{1}\right) . \mathrm{u}_{1}$ and $\mathrm{u}_{\mathrm{n}}$ are the end vertices of edge $E$ and $v_{i}, 2 \leq i \leq n-1$ are the middle vertices of $E$.

Definition 2.7. [5] A directed semigraph or disemigraph $D$ is a finite set of objects called vertices together with a set of ordered $n$-tuples of distinct vertices of $D$ for various $n \geq 2$ called directed edges or arcs, satisfying the condition $C$ given below. Suppose $a=\left(u_{1}, u_{2}\right.$, ...., $u_{n}$ ) is an arc.

Then for $1 \leq \mathrm{i}<\mathrm{j} \leq \mathrm{n}, u_{i}$ is adjacent to $u_{j}$ and $u_{j}$ is adjacent from $u_{i}$. Thus each $u_{i}$ is adjacent to $u_{j}, 1 \leq \mathrm{i}<\mathrm{j} \leq \mathrm{n}$ and each $u_{j}$ is adjacent from $u_{i}, 1 \leq \mathrm{i}<\mathrm{j} \leq \mathrm{n}$. $C$ : for any two distinct vertices $\mathrm{u}$ and $\mathrm{v}$ in a disemigraph $D$ there is at most one arc containing $u$ and $v$ 
A Study on the Normed Graphs and Disemigraphs

such that $\mathrm{u}$ is adjacent to $\mathrm{v}$ and at most one arc containing $u$ and $v$ such that $v$ is adjacent to $u$.

In a semigraph $G$ a vertex $v$ and an edge $E$ are incident to each other if $v \in E$ then $v$ and $E$ are said to cover each other.In a semigraph a set $S$ of vertices which cover all the edges is a vertex cover for $G$ and $S$ is an e-vertex cover if all the vertices in $S$ are end vertices of edges. Vertex covering number $\alpha_{0}=\alpha_{0}(G)$ is the minimum cardinality of a vertex cover. e-vertex covering number is denoted by $\alpha_{e}=\alpha_{e}(G)$.

The edge covering number $\alpha_{l}=\alpha_{l}(G)$ of $\mathrm{G}$ is the minimum cardinality of an edge cover. A set $S \subseteq V$ which covers all the 2- partitions of partial edges of $G$ is a ca-vertex cover for $\mathrm{G}$. The ca-vertex covering number $\alpha_{c a}=\alpha_{c a}(G)$ is the minimum cardinality of a ca-vertex cover of $G$.

The independence number $\beta_{0}=\beta_{0}(G)$ of $G$ is the maximum cardinality of an independent set of vertices of $G$. The $c a$-independence number $\beta_{\mathrm{ca}}=\beta_{c a}(G)$ of $G$ is the maximum cardinality of a $c a$-independent set of vertices of $G$.

There are many semigraph parameters $[2,3,4,8]$ which can be used to study the semigraphs. Of these we consider here the parameter vertex covering number which satisfies the conditions of norms in this study.

Proposition 2.6. [3] Let $G(V, X)$ be any semigraph with $p$ vertices and $q$ edges then $\alpha_{c a}+$ $\beta_{\mathrm{ca}}=p$.

There are different graphs associated with a given semigraph. The end vertex graph $G_{e}$, in which two vertices are adjacent if thye are end vertices in the semigraph. The adjacency graph $G_{a}$, in which two adjacent vertices in the semigraph are adjacent. The consecutive graph $G_{c a}$ in which the consecutive vertices are adjacent.

We consider the consecutive adjacency graph.

\section{The relative normed graph of a semigraph}

Definition 3.1. Any function defined on the set $S$ of spanning subsemigraphs of a semigraph is called a semigraph function. A semigraph function $g$ which satisfies the conditions of norm is said to be a semigraph norm or a norm defined on a semigraph.

Definition 3.2. Let $G$ be a semigraph and $S=\left\{H_{1}, H_{2}, . . H_{\mathrm{n}}\right\}$ be set of spanning subsemigraphs of $G$ then if $g$ is a norm defined on $G$ then we get the norm relative digraph of $G$ denoted by $N_{G}(S)$. The norm relative graph is always a digraph.

To construct $\mathbf{N}_{\mathbf{G}}(\mathbf{S})$ : Let $G$ be a semigraph and $S=\left\{H_{1}, H_{2}, . . H_{\mathrm{n}}\right\}$ be set of spanning subsemigraphs of $G$ and let $g$ be a norm defined on $G$. The vertex set of $N_{G}(S)$ is $S$ and the adjacency relation is, $H_{i}$ is adjacent to $H_{j}$ if $g\left(H_{i}\right) \leq g\left(H_{i}\right)$, for every $i$ and $j, i \neq j$, $H_{i}, H_{j} \in S$. Then $H_{i}$ is the tail and $H_{j}$ is the head. The resultant graph is the norm relative digraph of $G$ with respect to $S$.

Example 3.3. Consider Fig 1 and Fig 2. Let $\mathrm{G}$ be the semigraph and $\mathrm{S}=\left\{\mathrm{H}_{1}, \mathrm{H}_{2}, \mathrm{H}_{3}, \mathrm{H}_{4}\right\}$ be a set of spanning subsemigraphs then $N_{G}(S)$ is as given below. 
Mathew Varkey T.K and Susan Ray Joseph

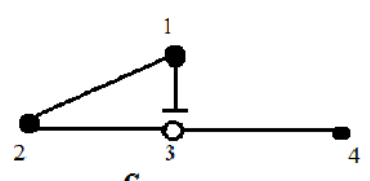

$\mathbf{G}$

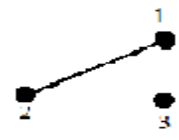

$\mathrm{H} \pm$

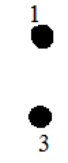

$\mathrm{H}_{1}$

Figure 1:

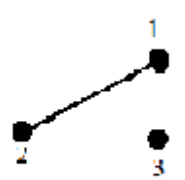

$\mathrm{H}$

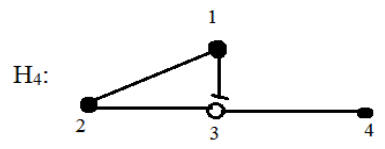

From the figures above the $\alpha_{c a}\left(\mathrm{H}_{1}\right)=0, \alpha_{c a}\left(\mathrm{H}_{2}\right)=1, \alpha_{c a}\left(\mathrm{H}_{3}\right)=1$ and $\alpha_{c a}\left(\mathrm{H}_{4}\right)=2$.

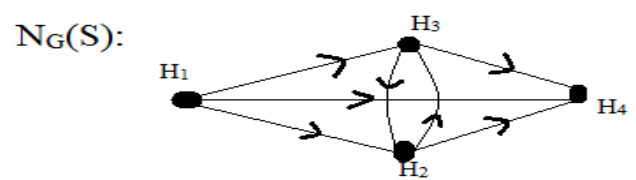

Figure 2:

Proposition 3.4. In the relative norm graph the degree of each vertex is $|\mathrm{S}|-1$.

Proof: The vertices of relative norm graph are spanning subsemigraphs and the norm function assigns a real value to each vertex. The real numbers are totally ordered hence any two vertices are adjacent.

Proposition 3.5. In a relative norm graph there is one vertex with outdegree $|\mathrm{S}|-1$ and another vertex with indegree $|S|-1$.

Proof: Since norm $g$ is a real valued function $g\left(H_{i}\right)$ is minimum $g\left(H_{i}\right) \leq g\left(H_{i}\right)$, for every $i$ and $j, \quad i \neq j, H_{i}, H_{j} \in S$. Hence there is one vertex with outdegree $|S|-1$. Similarly there exist one vertex with maximum norm function value, for that vertex indegree is $|S|-1$.

Example 3.6. Let $\mathrm{G}$ be the disemigraph and $\mathrm{S}=\left\{\mathrm{H}_{1}, \mathrm{H}_{2}, \mathrm{H}_{3}\right\}$ be a set of spanning subdisemigraphs then $\mathrm{N}_{\mathrm{G}}(\mathrm{S})$ is as given below in Fig:3 .

$\mathrm{G}:$

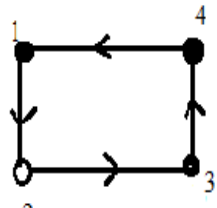

2

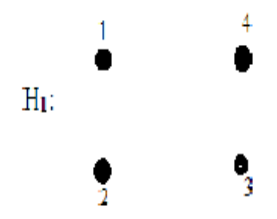

$V_{G}(\mathrm{~S}):$

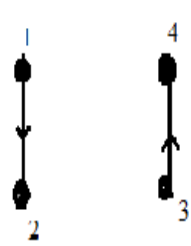

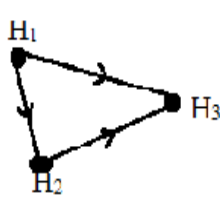

Figure 3:

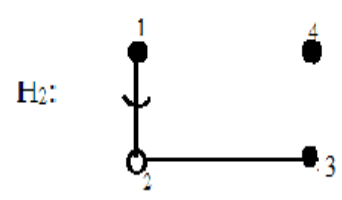

$G_{c a}:$

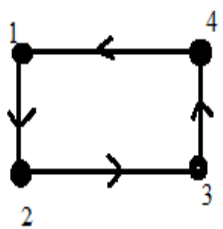




\section{A Study on the Normed Graphs and Disemigraphs}

To consider the normed graph of a disemigraph. We consider the consecutive adjacency graph $\mathrm{G}_{c a}$ of the disemigraph.

Definition 3.7. The graph $\mathrm{N}_{\mathrm{G}}(\mathrm{S})$ is a normed graph of a disemigraph if $N_{G}(S)=G_{c a}$. The example given below Fig 4 is a normed disemigraph.

Example 3.8. Let $\mathrm{G}$ be the disemigraph and $\mathrm{S}=\left\{\mathrm{H}_{1}, \mathrm{H}_{2}, \mathrm{H}_{3}, \mathrm{H}_{4}\right\}$ be a set of spanning subdisemigraphs. $\mathrm{N}_{\mathrm{G}}(\mathrm{S})$ and $\mathrm{G}_{\mathrm{ca}}$ are given below. Also $\mathrm{N}_{\mathrm{G}}(\mathrm{S}) \cong G_{c a}$

G:

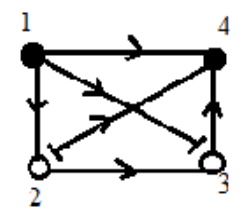

$\mathrm{H}_{2}$ :

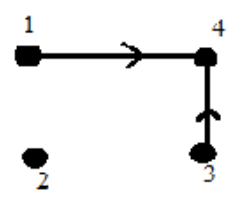

$\mathrm{N}_{\mathrm{G}}(\mathrm{S})$ :

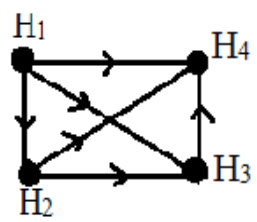

$\mathrm{H}_{1}$ :
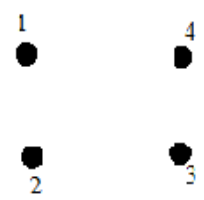

$\mathrm{H}_{4}$

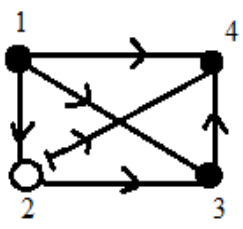

$\mathrm{H}_{3}$ :

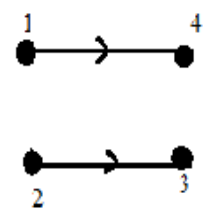

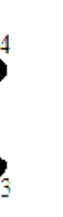

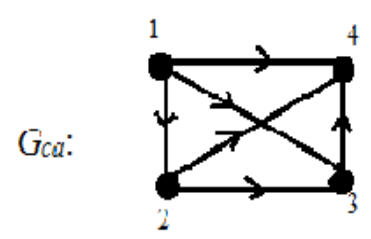

Figure 4:

\section{Remarks 3.9.}

(1) A disemigraph with a normed graph with respect to some semigraph norm need not be a normed graph for some other semigraph norm with the same adjacency relation.

(2) A disemigraph with a normed graph with respect to some semigraph norm need not be a normed graph with a different adjacency relation.

Propositions 3.10. The normed graph of a disemigraph are simple.

Proof: The normed graphs are simple by definition hence normed graphs of disemigraphs are simple.

Propositions 3.11. The collection of spanning subsemigraphs of a semigraph does not form a vector space.

Proof: The complement of a subsemigraph with respect to ring sum is not uniquely defined. Hence the collection of spanning subsemigraphs of a semigraph forming a vector space is not unique. Therefore does not form a vector space. 
Mathew Varkey T.K and Susan Ray Joseph

\section{Conclusion}

The norm function defined on digraph in [7] is applied on the set of spanning subdigraphs of a digraph in two ways. One on a set of spanning subdigraphs generating the normed graph and the other on the collection of spanning subdigraphs of a digraph forming a vector space. On considering the disemigraphs since the complement of a subdisemigraph is not unique the vector space of spanning subdisemigraphs cannot be defined uniquely.

\section{REFERENCES}

1. B.V.Limaye, Functional Analysis ( $2^{\text {nd }}$ edition $), 1996$.

2. K.Das, Algorithms to find wiener index of some graphs, Annals of Pure and Applied Mathematics, 7(1) (2014) 13-18.

3. S.S.Kamath and R.S.Bhat, Domination in semigraphs, preprint to Elsevier Science, September 2003.

4. S.V.Padmavathi, relation between convexity number and independence of graphs, Annals of Pure and Applied Mathematics, 9 (1) (2015) 9-12.

5. E.Sampathkumar, Semigraphs and their applications, Report on the Department of Science and Technology, project submitted to DST, India, May 2000.

6. K.S.Sriprabha, Normed linear spaces associated with graphs, Summaries of Ph.D thesis awarded by University of Kerala, 2015. http://iqac.keralauniversity.ac.in/docs/PhD_Summaries_V4-Final.pdf

7. G.Suresh Singh and K.S.Sriprabha, A note on normed graphs and its properties, Bulletin of Kerala Mathematics Association, 19(2) (2012) 319-336.

8. D.K.Thakkar and A.A.Prajapati, vertex covering and independence in semigraph, Annals of Pure and Applied Mathematics, 4 (2) (2013) 172-181. 